\title{
Energy Balance of a Bose Gas in a Curved Spacetime
}

\author{
Tonatiuh Matos ${ }^{*}$ Ana Avilez \\ Departamento de Física, Centro de Investigación y de Estudios Avanzados del IPN, \\ A. P. 14-740, 07000, Ciudad de México, México \\ Pierre-Henri Chavanis\$ \\ Laboratoire de Physique Théorique, Université Paul Sabatier, \\ 118 route de Narbonne 31062 Toulouse, France
}

\begin{abstract}
We derive a general energy balance equation for a self-interacting boson gas at vanishing temperature in a curved spacetime. This represents a first step towards a formulation of the first law of thermodynamics for a scalar field in general relativity. By using a $3+1$ foliation of the spacetime and performing a Madelung transformation, we rewrite the Klein-Gordon-Maxwell equations in a general curved spacetime into its hydrodynamic version where we can identify the different energy contributions of the system and separate them into kinetic, quantum, electromagnetic, and gravitational.

PACS numbers: 67.85.Hj, 67.85.Jk, 05.30.Jp, 04.40.Nr, 03.70.+k, 11.10.-z, 11.30.-j
\end{abstract}

Introduction. One of the most interesting open problems in general relativity is to identify different energy contributions in some relativistic objects, like neutron stars or black holes, since the standard laws of thermodynamics are not applicable in these cases. Essentially the problem stems from the fact that the spacetime metric describes both the geometrical structure and the dynamical aspects of the gravitational field [1]. In this Letter, as a first step, we address this problem for a charged boson gas at $T=0$ described by the Klein-Gordon-Maxwell (KGM) equations in a general curved spacetime. We decompose the metric according to the ADM $3+1$ formalism and carry out a Madelung transformation on the Klein-Gordon (KG) equation to derive the hydrodynamic equations governing the boson gas dynamics. In this way, it is easy to identify the different contributors to the total energy of the system through a balance equation that is an antecedent of the first law of thermodynamics in a curved spacetime.

Scalar fields are ubiquitous in modern physics, from the Higgs scalar that gives mass to matter particles at fundamental scales, up to huge astronomical and cosmological scales where they are used to model dark matter and dark energy. In most physical systems of interest, the dynamics of these scalars is well described by the original KGM equations, which are Lorentz and $U(1)$ invariant. It is well known that the KG modes can be interpreted as a set of independent bosonic particles living in a Minkowski spacetime. These bosons can be endowed with charge if a complex field is considered whose electromagnetic interaction is mediated by a gauge vector field. This is mathematically achieved by promoting the global $U(1)$ symmetry to a local one. Furthermore, the self-interactions between fields are encoded in a potential $V(\Phi)$.

Although all this KG machinery has been the basis of great achievements, further developments of this framework are needed to model phenomena beyond the Minkowskian threshold. In this work, we are interested in solutions of the KG equation as models of objects at large scales like compact stars and dark matter halos.

The first attempt to describe astronomical objects as macroscopic bosonic states was made by Wheeler [2] who aimed at constructing stable particle-like solutions from classical electromagnetic fields coupled to general relativity. However, his solutions were unstable. Later, Kaup [3] and Ruffini and Bonazzola [4] introduced the notion of boson stars that could be useful to model compact stars having certain advantages over fluid neutron star models [5]. More recently, scalar fields as dark matter were suggested as a set of bosonic modes all laying in the ground state making up a macroscopic wave function which corresponds to a galactic halo [6 9].

In the non-relativistic limit, the KG equation endowed with a self-interaction reduces to the Gross-Pitaevskii (GP) equation. Since it is based on Newtonian gravity, the GP equation cannot model compact objects. However, it is possible to construct a more suitable framework by extending the GP equation to curved spacetimes. The main goal of this work is, first, to generalize the GP equation in order to model a charged boson gas laying in a curved spacetime. Secondly, we use the hydrodynamic representation of the complex scalar field equations in terms of the Madelung variables to derive a conservation equation that can be interpreted as the energy balance of the different components involved. We also derive its Newtonian limit.

Field Equations. In what follows we model the boson gas as a set of excitations of a self-interacting, charged, complex scalar field which is minimally coupled to a gauge vector field mediating the electromagnetic interaction. Gravity is interpreted as a geometrical phenomenon, i.e. the surrounding spacetime of a massive body acquires curvature as described by General Rela- 
tivity. We shall not deal explicitly with the Einstein field equations but we consider an arbitrary spacetime geometry. We aim to extend the KGM equations, with local $U(1)$ symmetry, by using coordinates for a 4-dimensional manifold playing the role of the physical curved spacetime whose geometry is encoded by a metric $g$. From hereafter we use the units $c=\hbar=\epsilon_{0}=\mu_{0}=1$, for $c$ the speed of light, $\hbar$ the reduced Planck constant, $\epsilon_{0}$ and $\mu_{0}$ the permittivity and permeability of free space, respectively. We define the electromagnetic d'Alembert operator as $\square_{\mathrm{E}}=\left(\nabla^{\mu}+i e A^{\mu}\right)\left(\nabla_{\mu}+i e A_{\mu}\right)$, where $e$ is the charge unit, and $A_{\mu}$ is the $U(1)$ gauge vector field corresponding to the Maxwell 4-potential, such that the KGM equations are given by

$$
\begin{aligned}
& \square_{\mathrm{E}} \Phi-\frac{\mathrm{d} V}{\mathrm{~d} \Phi^{*}}=0, \\
& \nabla_{\nu} F^{\nu \mu}=J^{E \mu},
\end{aligned}
$$

for the complex scalar field $\Phi(t, \mathbf{x})$ and its complex conjugate $\Phi^{*}(t, \mathbf{x})$. The Faraday tensor is given by

$$
F_{\mu \nu}=\nabla_{\mu} A_{\nu}-\nabla_{\nu} A_{\mu},
$$

and the conserved 4-current is defined as

$$
J_{\mu}^{E} \equiv i \frac{e}{2 m^{2}}\left[\Phi\left(\nabla_{\mu}-i e A_{\mu}\right) \Phi^{*}-\Phi^{*}\left(\nabla_{\mu}+i e A_{\mu}\right) \Phi\right] .
$$

We introduce scalar self-interactions by using the doublewell (Mexican-hat) self-interacting potential

$$
V=m^{2}|\Phi|^{2}+\frac{\lambda}{2}|\Phi|^{4}
$$

describing a system of bosonic excitations which condense into a single macroscopic ground state.

We use the $3+1$ foliation of the metric [10, 11] so that the line-element reads

$$
\mathrm{d} s^{2}=-N^{2} c^{2} \mathrm{~d} t^{2}+\gamma_{i j}\left(\mathrm{~d} x^{i}+N^{i} c \mathrm{~d} t\right)\left(\mathrm{d} x^{j}+N^{j} c \mathrm{~d} t\right),
$$

where $N$ is the lapse function which measures the proper time of the observers traveling along the world line, $N^{i}$ is the shift vector that measures the displacement of the observers between the spatial slices, and $\gamma_{i j}$ is the spatial metric.

Generalized Gross-Pitaevskii Equation. Here we show that the KG equation with the hat potential can be transformed into a relativistic GP equation. The nonrelativistic GP equation, which is a nonlinear version of Schrödinger's equation, has been of great interest in quantum mechanics and statistical mechanics since it accounts for correlations between quantum particles. An important application of this framework is the study of superfluidity and phase transitions. Bogolyubov 12 first tried to model superfluidity as an imperfect BEC due to the weak repulsion between the bosons. It is worth noting that, in contrast to the standard GP equation for neutral particles in a spatially flat spacetime, charged bosons in a curved spacetime find interesting applications in the context of dark matter, boson stars and neutron stars with superfluid cores.

An important mathematical feature of the KG equation is that, in some circumstances, it admits nondispersive solutions, as those relevant in scattering processes. The Derrick theorem states that no-regular, static, non-topological localized scalar field solutions are stable in a spatially flat space [13]. This constraint is avoided by adopting a harmonic decomposition for the complex scalar field,

$$
\Phi(t, \mathbf{x})=\Psi(t, \mathbf{x}) \exp \left(-i \omega_{0} t\right),
$$

where $\omega_{0}$ is a constant that can be either the mass or the frequency of massless particles. Although the field is non static, the spacetime remains static and then the KG equation admits soliton-like solutions [13, 14]. By plugging such field-redefinition into the KG equation (1) we obtain

$$
\begin{aligned}
& i \nabla^{0} \Psi-\frac{1}{2 \omega_{0}} \square_{E} \Psi+\frac{1}{2 \omega_{0}}\left(m^{2}+\lambda n\right) \Psi \\
& +\frac{1}{2}\left(-\frac{\omega_{0}}{N^{2}}-2 e A^{0}+i \square t\right) \Psi=0,
\end{aligned}
$$

where $n(t, \mathbf{x}) \equiv|\Phi|^{2}=|\Psi|^{2}$ is defined as the scalar field density and $\square t=\nabla^{\mu} \nabla_{\mu} t .{ }^{1}$ Equation (8) is the KG equation in terms of $\Psi$ and we name it the generalized GP equation in curved spacetimes for the potential (5).

Hydrodynamic Representation. The hydrodynamic approach for the Schrödinger equation was introduced by Madelung [15] (see also Bohm [16, 17]), who showed that it is equivalent to Euler equations for an irrotational fluid with an additional quantum potential. The boson gas that we study can be interpreted as a real fluid described by the quantum Euler equations. This hydrodynamic representation has been widely used in the literature: 18 21] used it for BEC dark matter, 22] for relativistic BECs, including electromagnetic interactions by [23], and in the case of relativistic BEC stars by [24, 25]. In this section we derive the hydrodynamic representation for the generalized GP equation (8). We carry out the following Madelung transformation

$$
\Phi(t, \mathbf{x})=\sqrt{n} \exp (i \theta)=\sqrt{n} \exp \left[i\left(S-\omega_{0} t\right)\right]
$$

where the amplitude $\Psi$ is decomposed into a density $n(t, \mathbf{x})$ and a phase $S(t, \mathbf{x})$ encoding the geometry and

\footnotetext{
${ }^{1}$ In order to define a slicing condition to calculate the lapse function $N$, a common approach uses harmonic coordinates, defined by asking for the wave operator to vanish: $\square x^{\alpha}=0$. In particular, for $\square t=0$ the harmonic slicing condition on the lapse function is obtained. Here we do not assume any specific foliation condition.
} 
evolution of the front-wave solution. In this way, the KG/GP equation splits into its imaginary and real parts respectively,

$$
\begin{gathered}
\nabla_{\mu} \sqrt{n}\left(2 \nabla^{\mu} \theta+e A^{\mu}\right)+e \nabla_{\mu}\left(A^{\mu} \sqrt{n}\right)+\sqrt{n} \square \theta=0, \\
\square \sqrt{n}-\sqrt{n}\left[\nabla_{\mu} \theta\left(\nabla^{\mu} \theta+2 e A^{\mu}\right)+e^{2} A^{2}+m^{2}+\lambda n\right]=0,
\end{gathered}
$$

where $A^{2}=A^{\mu} A_{\mu}$. After applying the Madelung transformation, the current (4) turns into

$$
J^{E}{ }_{\mu}=\frac{n e}{m^{2}}\left(\nabla_{\mu} \theta+e A_{\mu}\right) .
$$

Interestingly, a relativistic quantum particle in a flat spacetime with an electromagnetic field has a mechanical momentum $m \mathbf{u}=\mathbf{p}-e \mathbf{A}$, with $\mathbf{p}$ the canonical momentum and $\mathbf{A}$ the magnetic vector potential. By writing its wave function in hydrodynamic variables, it results that $\mathbf{p}$ is equal to the gradient of the phase of the wave function. In a similar way for our boson gas, the electromagnetic 4-momentum corresponds to the sum of individual mechanical momenta, namely $J^{E}{ }_{\mu}=(e / m) n u_{\mu}$. In terms of $J^{E}{ }_{\mu}$, Eqs. (10) and (11) read

$$
\begin{aligned}
\nabla^{\mu} J^{E}{ }_{\mu} & =0, \\
J^{E}{ }_{\mu} J^{E \mu}+\frac{n^{2} e^{2}}{m^{4}}\left(m^{2}+\lambda n-\frac{\square \sqrt{n}}{\sqrt{n}}\right) & =0 .
\end{aligned}
$$

Then, by interpreting the KG equation as a generalrelativistic GP equation, through the Madelung transformation, it splits into the continuity (13) and quantum Hamilton-Jacobi (14) equations above. Such quantum version of the Hamilton-Jacobi equation differs from the classical one only by the last term on the left-hand side of Eq. (14), which corresponds to the de Broglie [26] relativistic quantum potential.

Now, let us take the continuity equation (13) and notice that

$$
\int_{\mathbb{V}} \nabla_{\mu} J^{E \mu} \mathrm{d} V=\int_{\mathbb{V}} \nabla_{0} J^{E 0} \mathrm{~d} V+\int_{\mathbb{S}} k_{j} J^{E j} \mathrm{~d} S=0,
$$

where $\mathbb{S}$ is an arbitrary surface enclosing the volume $\mathbb{V}$ containing the system and $k^{j}$ is the vector orthogonal to $\mathbb{S}$. We assume that far away from the sources $J^{E}{ }_{j}$ is negligible, so we are free to choose a volume $\mathbb{V}$ such that the surface integral of Eq. (15) vanishes. Thus, the quantity $Q=\int_{\mathbb{V}} J^{E 0} \mathrm{~d} V$ is a conserved charge,

$$
\frac{\mathrm{d} Q}{\mathrm{~d} t}=\int_{\mathbb{V}} \nabla_{0} J^{E 0} \mathrm{~d} V=0 .
$$

In conclusion, the continuity equation (13) expresses the conservation of the charge of the scalar field.
Continuity and Euler Equations. Let us define the "velocity" $v_{\mu}$ of an individual particle as

$$
m v_{\mu} \equiv \nabla_{\mu} S+e A_{\mu} .
$$

It is important to mention that $v_{\mu}$ is not a 4 -vector strictly speaking. We also stress that we are working in a frame in which the contribution of the rest mass energy has been subtracted from the quadrivelocity. In terms of $v_{\mu}$, the continuity and quantum Hamilton-Jacobi equations (13) and (14) become

$$
\begin{aligned}
\nabla^{\mu}\left(n v_{\mu}\right)-\frac{\omega_{0}}{m}\left(\nabla^{0} n+n \square t\right) & =0, \\
v_{\mu} v^{\mu}-\frac{2 \omega_{0}}{m} v^{0}-\frac{\omega_{0}^{2}}{m^{2} N^{2}}+1+\frac{\lambda}{m^{2}} n-\frac{1}{m^{2}} \frac{\square \sqrt{n}}{\sqrt{n}} & =0 .
\end{aligned}
$$

Equation (18) governs the evolution of the density of the boson gas whilst Eq. (19) governs the evolution of its phase.

After applying the covariant derivative $\nabla_{\alpha}$ to Eq. (19), using the Leibniz rule to the first two terms, and using Maxwell's equation (2), we obtain the Euler equation

$$
\begin{aligned}
& v_{\mu} \nabla^{\mu} v_{\alpha}-\frac{\omega_{0}}{m} \nabla^{0} v_{\alpha}-\frac{\omega_{0}^{2}}{2 m^{2}} \nabla_{\alpha}\left(\frac{1}{N^{2}}\right) \\
+ & \frac{\lambda}{2 m^{2}} \nabla_{\alpha} n-\frac{1}{2 m^{2}} \nabla_{\alpha}\left(\frac{\square \sqrt{n}}{\sqrt{n}}\right) \\
+ & \frac{e}{m}\left[v_{\mu}\left(\nabla_{\alpha} A^{\mu}-\nabla^{\mu} A_{\alpha}\right)-\frac{\omega_{0}}{m}\left(\nabla_{\alpha} A^{0}-\nabla^{0} A_{\alpha}\right)\right]=0 .
\end{aligned}
$$

At this point, we can identify different analogues to physical quantities. In first place, we spot the covariant definition of the Lorentz force in a curved spacetime, given by

$$
F_{\alpha}^{E} \equiv-\frac{e}{m}\left(v_{\mu} F_{\alpha}{ }^{\mu}-\frac{\omega_{0}}{m} F_{\alpha}^{0}\right) .
$$

In second place, we identify the gravitational "force" which, in spite of the geometrical nature of gravity assumed here, is an actual measurement of the curvature associated with the gravitational strength quantified by the time-time component of the metric

$$
F_{\alpha}^{G} \equiv-2 \nabla_{\alpha} U^{G}, \quad U^{G} \equiv-\frac{\omega_{0}^{2}}{4 N^{2} m^{2}},
$$

where $U^{G}$ is the gravitational "potential" contribution due to the metric time-component related to the gravitational strength. In third place, the quantum force is given by

$$
F^{Q}{ }_{\alpha} \equiv-\nabla_{\alpha} U^{Q}, \quad U^{Q} \equiv-\frac{1}{2 m^{2}} \frac{\square \sqrt{n}}{\sqrt{n}},
$$

where $U^{Q}$ is the quantum potential. Finally, a measure of the temporal and spatial variations of the density of the scalar field is characterized by

$$
F_{\alpha}^{n} \equiv-\nabla_{\alpha} h, \quad h \equiv \frac{\lambda n}{2 m^{2}},
$$


where $h$ is the enthalpy. For future purposes, we introduce the pressure $p \equiv \lambda n^{2} / 4 m^{2}$ which satisfies the Gibbs-Duhem relation $d h=d p / n$ and the internal energy $U^{n}=\lambda n / 4 m^{2}$ which satisfies the local law $d U^{n}=$ $-p d(1 / n)$. Notice that $F^{n}{ }_{\alpha}$ is only present when the self-interactions are turned-on $(\lambda \neq 0)$.

In summary, if all the previous quantities (21)-(24) are plugged into Eq. (20), we obtain

$$
-\frac{\omega_{0}}{m} \nabla^{0} v_{\alpha}+v_{\mu} \nabla^{\mu} v_{\alpha}=F_{\alpha}^{E}+F_{\alpha}^{G}+F_{\alpha}^{Q}+F_{\alpha}^{n} .
$$

Equations (18), (19) and (25) are dynamically equivalent to the KGM equations. However, written in terms of the $n$ and $v_{\mu}$ variables, they give rise to a different physical interpretation. They may be viewed as the generalized continuity, Hamilton-Jacobi, and Euler hydrodynamic equations.

Balance Equation. We now derive the different energy contributions for the system of charged bosons and the total energy balance equation. Contracting Eq. (25) with $n v_{\alpha}$ and using the Leibniz rule, we get

$\nabla^{\mu}\left(v_{\mu} n K\right)-\frac{\omega_{0}}{m} \nabla^{0}(n K)=n v^{\mu}\left(F_{\mu}^{E}+F_{\mu}^{G}+F_{\mu}^{Q}+F_{\mu}^{n}\right)$,

where $K=v_{\alpha} v^{\alpha} / 2$ is defined as the kinetic energy per unit of mass.

In order to express the electromagnetic contribution in terms of the symmetric energy-momentum tensor, it is convenient to introduce the current of charge

$$
J^{E \mu}=\frac{e}{m} n\left(v^{\mu}-\frac{\omega_{0}}{m} \nabla^{\mu} t\right) .
$$

The Lorentz force (21) takes the form

$$
F_{\alpha}^{E}=-\frac{1}{n} J^{E \mu} F_{\mu \alpha} .
$$

Using the covariant Maxwell equations (2), after some tensor algebra and using the Jacobi identity of the Riemann tensor, we obtain

$$
F^{E \alpha}=\frac{1}{n} \nabla_{\beta} \Theta^{\alpha \beta}
$$

where the symmetric electromagnetic energy-momentum tensor is

$$
\Theta^{\alpha \beta} \equiv g^{\alpha \mu} F_{\mu \nu} F^{\nu \beta}+\frac{1}{4} g^{\alpha \beta} F^{\mu \nu} F_{\mu \nu} .
$$

Its components $\left\{\Theta^{00}, \Theta^{0 i}\right\}=\left\{U^{E}, P_{i}^{E}\right\}$ are the generalized electromagnetic energy density and the Poynting vector, respectively. On the other hand, using Eq. (27) the first term in the r.h.s. of Eq. (26) reads

$$
n v^{\alpha} F_{\alpha}^{E}=\left(\frac{m}{e} J^{E \alpha}+\frac{\omega_{0}}{m} n \nabla^{\alpha} t\right) F_{\alpha}^{E} .
$$

The antisymmetry of the Faraday tensor implies that $J^{E \alpha} F_{\alpha}^{E} \propto J^{E \alpha} J^{E \mu} F_{\mu \alpha}=0$ which leads us to obtain from Eqs. (29) and (31):

$$
n v^{\alpha} F_{\alpha}^{E}=-\frac{\omega_{0}}{m} n F^{E 0}=-\frac{\omega_{0}}{m} \nabla_{\alpha} \Theta^{\alpha 0} .
$$

Since Eq. (32) is a gauge invariant expression, the tensor structure of our results below will not be affected by the gauge fixing procedure used on the electromagnetic sector.

Now, using the continuity equation (18) and the Leibniz rule, it is easy to get the following relation for an arbitrary field $U$ :

$$
\begin{aligned}
- & \frac{\omega_{0}}{m} \nabla^{0}(n U)+\nabla^{\mu}\left(v_{\mu} n U\right)-n v_{\mu} \nabla^{\mu} U \\
+\frac{\omega_{0}}{m} n \nabla^{0} U & +\frac{\omega_{0}}{m} n U \square t=0,
\end{aligned}
$$

which can be applied to the internal energy $U^{n}$, the quantum potential $U^{Q}$ and the gravitational contribution $U^{G}$.

A further simplification is made by noting that

$$
n \nabla^{0} U^{Q}=-\frac{1}{4 m^{2}} \nabla_{\mu}\left[n \nabla^{0}\left(\nabla^{\mu} \ln n\right)\right]
$$

This relation along with Eq. (33) leads to

$$
\begin{aligned}
- & \frac{\omega_{0}}{m} \nabla^{0}\left(n U^{Q}\right)+\nabla^{\mu}\left(n v_{\mu} U^{Q}+J_{\mu}^{Q}\right) \\
-n v_{\mu} \nabla^{\mu} U^{Q} & +\frac{\omega_{0}}{m} n U^{Q} \square t=0,
\end{aligned}
$$

where we have defined the quantum flux as

$$
J_{\mu}^{Q} \equiv-\frac{\omega_{0}}{4 m^{3}} n \nabla_{\mu}\left(\nabla^{0} \ln n\right) .
$$

If we sum Eqs. (26) and (33) for $U^{n}$ and $U^{G}$, and Eq. (36), we obtain

$$
\begin{aligned}
& -\frac{\omega_{0}}{m} \nabla^{0}\left(n \mathcal{U}^{s}\right)+\nabla^{\mu}\left(n v_{\mu} \mathcal{U}^{s}\right)+\frac{\omega_{0}}{m} \nabla^{i} P^{E}{ }_{i}+\frac{\omega_{0}}{m} \nabla^{0} U^{E} \\
& +\nabla^{\mu}\left(J^{Q}{ }_{\mu}+p v_{\mu}\right)+\frac{\omega_{0}}{m} n \nabla^{0} U^{G}+n v^{\mu} \nabla_{\mu} U^{G} \\
& +\frac{\omega_{0}}{m} n\left(U^{G}+U^{Q}\right) \square t=0,
\end{aligned}
$$

where we have introduced the energy density of the scalar field $\mathcal{U}^{s} \equiv K+U^{G}+U^{Q}+U^{n}$. Equation (38) is the total energy balance equation. The total flux associated with the energy density of the scalar field $\mathcal{U}^{s}$ involves the energy flux $n v_{\mu} \mathcal{U}^{s}$, the quantum flux $J^{Q}{ }_{\mu}$, the pressure flux $p v_{\mu}$, and a contribution due to the gravitational interaction $U^{G}$. The flux associated with the electromagnetic energy density $U^{E}$ is the Poynting vector $P^{E}{ }_{i}$.

Newtonian Limit. In the longitudinal Newtonian gauge in flat space

$$
\mathrm{d} s^{2}=-(1-2 \varphi) c^{2} \mathrm{~d} t^{2}+\delta_{i j}(1+2 \varphi) \mathrm{d} x^{i} \mathrm{~d} x^{j} .
$$


Within the non relativistic limit, Eq. (8) becomes the traditional GP equation [27] and the hydrodynamic equations (18), (19) and (25) reduce to

$$
\begin{gathered}
\partial_{t} n+\nabla \cdot(n \mathbf{v})=0 \\
\partial_{t} S+\frac{1}{2 m}(\nabla S-e \mathbf{A})^{2}=-m\left(U^{Q}+h+\varphi+\frac{e}{m} \varphi_{E}\right),
\end{gathered}
$$$$
\partial_{t} \mathbf{v}+(\mathbf{v} \cdot \nabla) \mathbf{v}=-\nabla U^{Q}-\frac{1}{n} \nabla p-\nabla \varphi+\frac{e}{m}(\mathbf{E}+\mathbf{v} \times \mathbf{B}),
$$

where $U^{Q}=-\left(1 / 2 m^{2}\right) \Delta \sqrt{n} / \sqrt{n}$ is the Madelung 15. classical quantum potential and $(\mathbf{E}, \mathbf{B})=\left(-\partial_{t} \mathbf{A}-\right.$ $\left.\nabla \varphi_{E}, \nabla \times \mathbf{A}\right)$ is the electromagnetic field. Taking the scalar product of Eq. (42) with $\mathbf{v}$ and using the continuity equation (40), we obtain

$$
\begin{aligned}
\partial_{t}(n K)+\nabla(n K \mathbf{v})= & -n \mathbf{v} \cdot \nabla U^{Q}-\mathbf{v} \cdot \nabla p \\
& -n \mathbf{v} \cdot \nabla \varphi+\mathbf{J}^{E} \cdot \mathbf{E}
\end{aligned}
$$

where $K=v^{2} / 2$ is the density of kinetic energy and we have introduced the current of charge $\mathbf{J}^{E}=(e / m) n \mathbf{v}$. From the Maxwell equations $\nabla \times \mathbf{E}=-\partial_{t} \mathbf{B}$ and $\nabla \times \mathbf{B}=$ $\mathbf{J}^{E}+\partial_{t} \mathbf{E}$, using the identity $\nabla \cdot(\mathbf{E} \times \mathbf{B})=\mathbf{B} \cdot(\nabla \times \mathbf{E})-$ $\mathbf{E} \cdot(\nabla \times \mathbf{B})$, one can show that $\mathbf{J}^{E} \cdot \mathbf{E}=-\partial_{t} U^{E}-\nabla$. $\mathbf{P}^{E}$, where $U^{E}=\left(E^{2}+B^{2}\right) / 2$ is the electromagnetic energy and $\mathbf{P}^{E}=\mathbf{E} \times \mathbf{B}$ is the Poynting vector. From the continuity equation (40), we get

$$
\partial_{t}(n U)+\nabla(n U \mathbf{v})=n \partial_{t} U+n \mathbf{v} \cdot \nabla U
$$

Applying Eq. (44) to $U^{Q}, U^{n}$ and $U^{G}=\varphi / 2$, using the identity

$$
n \partial_{t} U^{Q}=-\nabla \cdot \mathbf{J}^{Q} ; \quad \mathbf{J}^{Q}=\frac{1}{4 m^{2}} n \partial_{t} \nabla \ln n,
$$

and introducing the energy density of the scalar field $\mathcal{U}^{s}=K+U^{Q}+U^{n}+U^{G}$, we obtain the local energy conservation equation

$$
\begin{aligned}
\partial_{t}\left(n \mathcal{U}^{s}\right) & +\nabla \cdot\left(n \mathcal{U}^{s} \mathbf{v}\right)+\partial_{t} U^{E}+\nabla \cdot \mathbf{P}^{E}+\nabla \cdot(p \mathbf{v}) \\
& +\nabla \cdot \mathbf{J}^{Q}+\frac{1}{2} n \mathbf{v} \cdot \nabla \varphi-\frac{1}{2} n \partial_{t} \varphi=0
\end{aligned}
$$

Using the Poisson equation: $\nabla^{2} \varphi=4 \pi G n$, and the continuity equation (40), one can easily show that Eq. (46) implies the global conservation of the total (scalar field + electromagnetic) energy $E_{\text {tot }}=\int\left(n \mathcal{U}^{s}+U^{E}\right) \mathrm{d} \mathbf{x}$, i.e. $\dot{E}_{\text {tot }}=0$.

This equation relates different components of the energy of a set of bosons laying within a gravitational potential well deforming the Minkowski spacetime. This approximation suffices to describe a host of astrophysical situations.

Discussion. In this Letter, we have derived a generalized hydrodynamic formulation of a system of charged bosonic excitations laying in a curved spacetime, which is governed by a general relativistic set of continuity, Hamilton-Jacobi, and Euler equations written up in Madelung variables, equivalent to the Klein-GordonMaxwell equations. Performing a $3+1$ foliation of the spacetime, we have shown that it is possible to split the total energy of the system into different contributions, specifically the kinetic, quantum and electromagnetic parts, and a term due to the gravitational field strength. The main result of this Letter is the energy balance equation for the boson gas, being a first step towards the first law of thermodynamics for a scalar field in general relativity. It is worth remarking that this result is general and has not been derived before. We believe that it is possible to carry out the same procedure by decomposing the matter equation for fermions.

This work was partially supported by CONACyT México under grants CB-2011 No. 166212, CB-201401 No. 240512, Project No. 269652 and Fronteras Project 281; Xiuhcoatl and Abacus clusters at Cinvestav, IPN; I0101/131/07 C-234/07 of the Instituto Avanzado de Cosmología (IAC) collaboration (http://www.iac.edu.mx/). We would like to acknowledge L.E. Padilla for useful comments and corrections in the elaboration of this manuscript. T.B. and A.A. acknowledge financial support from CONACyT postdoctoral fellowships.

* Electronic address: tmatos@fis.cinvestav.mx

$\dagger$ Electronic address: aavilez@fis.cinvestav.mx

‡ Electronic address: tbernal@fis.cinvestav.mx

$\S$ Electronic address: chavanis@irsamc.ups-tlse.fr

[1] R. M. Wald, General Relativity (University of Chicago, 1984).

[2] J. A. Wheeler, Phys. Rev. 97, 511 (1955).

[3] D. J. Kaup, Phys. Rev. 172, 1331 (1968).

[4] R. Ruffini and S. Bonazzola, Phys. Rev. 187, 1767 (1969).

[5] V. Faraoni, Phys. Rev. D 85, 024040 (2012).

[6] S.-J. Sin, Phys. Rev. D 50, 3650 (1994).

[7] F. S. Guzman, T. Matos, and H. Villegas-Brena, Rev. Mex. Astron. Astrofis. 37, 63 (2001).

[8] F. S. Guzmán and T. Matos, Classical and Quantum Gravity 17, L9 (2000).

[9] A. Suárez, V. H. Robles, and T. Matos, in A Review on the Scalar Field/Bose-Einstein Condensate Dark Matter Model in Accelerated Cosmic Expansion. Proceedings of the IV International Meeting on Gravitation and Cosmology.Astrophysics and Space Science Proceedings, edited by C. Moreno González, J. E. Madriz Aguilar, and L. M. Reyes Barrera (2014), vol. 38, p. 107.

[10] R. Arnowitt, S. Deser, and C. W. Misner, General Relativity and Gravitation 40, 1997 (2008).

[11] M. Alcubierre, Introduction to $3+1$ Numerical Relativity, International Series of Monographs on Physics (OUP Oxford, 2008), ISBN 9780191548291.

[12] N. N. Bogolyubov, J. Phys.(USSR) 11, 23 (1947), [Izv. Akad. Nauk Ser. Fiz.11,77(1947)]. 
[13] G. H. Derrick, Journal of Mathematical Physics 5, 1252 (1964).

[14] G. Rosen, Journal of Mathematical Physics 7, 2066 (1966).

[15] E. Madelung, Zeitschrift fur Physik 40, 322 (1927).

[16] D. Bohm, Phys. Rev. 85, 166 (1952).

[17] D. Bohm, Phys. Rev. 85, 180 (1952).

[18] C. G. Böhmer and T. Harko, JCAP 6, 025 (2007).

[19] P.-H. Chavanis, Phys. Rev. D 84, 043531 (2011).

[20] A. Suárez and P.-H. Chavanis, Phys. Rev. D 92, 023510 (2015).

[21] D. Bettoni, M. Colombo, and S. Liberati, JCAP 2, 004 (2014).

[22] S. Fagnocchi, S. Finazzi, S. Liberati, M. Kormos, and
A. Trombettoni, New Journal of Physics 12, 095012 (2010).

[23] P.-H. Chavanis and T. Matos, ArXiv e-prints (2016), 1606.07041.

[24] P.-H. Chavanis and T. Harko, Phys. Rev. D86, 064011 (2012).

[25] P.-H. Chavanis, The European Physical Journal Plus 130, 181 (2015).

[26] L. De Broglie, J. Phys. Radium 8, 225 (1927).

[27] L. Pitaevskii and S. Stringari, Bose-Einstein Condensation, International Series of Monographs on Physics (OUP Oxford, 2003), ISBN 780198507192. 